\title{
SOLVABILITY CRITERION AND REPRESENTATION OF SOLUTIONS OF $n$-NORMAL AND $d$-NORMAL LINEAR OPERATOR EQUATIONS IN A BANACH SPACE
}

\author{
V. F. Zhuravlev
}

UDC 517.983

\begin{abstract}
On the basis of a generalization of the well-known Schmidt lemma to the case of $n$-normal and $d$ normal linear bounded operators in a Banach space, we propose constructions of generalized inverse operators. We obtain criteria for the solvability of linear equations with these operators and formulas for the representation of solutions of these equations.
\end{abstract}

The investigation of the solvability of boundary-value problems for countable systems of differential equations [1] with finitely many boundary conditions and finite systems of differential equations with infinitely many boundary conditions and the construction of their solutions require the construction of generalized inverse operators for $n$-normal and $d$-normal operators [2].

The most completely studied and widely used methods for the generalized inversion of normally solvable operators are the Schmidt construction [3] for Fredholm operators (with nonzero kernels) and its analog for Noetherian operators [4].

In the present paper, we prove lemmas that generalize the Schmidt lemma [5] to the case of normally solvable operators that are $n$-normal or $d$-normal, propose constructions of their generalized inverses, and prove theorems on the solvability of linear equations with these operators in Banach spaces and on the representation of solutions.

\section{Statement of the Problem}

Consider the problem of finding conditions for the existence of solutions of the equation

$$
L x=y
$$

and their construction under the assumption that $L$ is a linear, bounded, normally solvable operator that acts from a Banach space $\mathbf{B}_{1}$ into a Banach space $\mathbf{B}_{2}, L: \mathbf{B}_{1} \rightarrow \mathbf{B}_{2}$, and is $n$-normal or $d$-normal. If $L: \mathbf{B}_{1} \rightarrow \mathbf{B}_{2}$ is a linear, bounded, $n$-normal operator, then we assume that its image is complementable in the space $\mathbf{B}_{2}$ [6], i.e.,

$$
\mathbf{B}_{2}=Y \oplus R(L)
$$

If $L: \mathbf{B}_{1} \rightarrow \mathbf{B}_{2}$ is a linear, bounded, $d$-normal operator, then its kernel $N(L)$ is complementable in the space $\mathbf{B}_{1}$, i.e.,

$$
\mathbf{B}_{1}=N(L) \oplus X
$$

Zhitomir National Agroecological University, Zhitomir, Ukraine.

Translated from Ukrains'kyi Matematychnyi Zhurnal, Vol. 62, No. 2, pp. 167-182, February, 2010. Original article submitted April 6, 2009; revision submitted November 27, 2009. 
Let $\operatorname{dim} N(L)=\mu$ and $\operatorname{dim} N\left(L^{*}\right)=v$ denote the dimensions of the null spaces of the operator $L$ and its adjoint $L^{*}$, respectively. According to S. Krein's classification [7], if $\mu$ is finite and $v$ is infinite, then $L$ is an $n$-normal operator, and, vice versa, if $\mu$ is infinite and $v$ is finite, then $L$ is a $d$-normal operator.

We consider the problem of constructing a bounded generalized inverse $L^{-}$of the operator $L$, finding conditions for the solvability of Eq. (1), and constructing a representation of its solutions.

\section{Auxiliary Statements}

First, we consider $n$-normal operators. Let $\left\{f_{i}\right\}_{i=1}^{\mu} \subset N(L), f_{i}=\operatorname{col}\left(f_{i}^{(1)}, f_{i}^{(2)}, f_{i}^{(3)}, \ldots\right)$, be a basis of the subspace $N(L)$, which exists because the latter is finite-dimensional $(\mu<\infty)$. Assume that the space $\mathbf{B}_{2}$ has a basis. The space $\mathbf{B}_{2}^{*}$ also has a basis as a space dual to $\mathbf{B}_{2}$ [8, p. 131]. Therefore, the subspace $N^{*}(L) \subset \mathbf{B}_{2}^{*}$ has a complete system of basis elements (functionals) $\left\{\varphi_{s}(\cdot)\right\}_{s=1}^{\infty} \subset N^{*}(L)$, $\varphi_{s}(\cdot)=\operatorname{col}\left(\varphi_{s}^{(1)}(\cdot), \varphi_{s}^{(2)}(\cdot), \varphi_{s}^{(3)}(\cdot), \ldots\right)$. For the elements $\left\{f_{i}\right\}_{i=1}^{\mu}$ and functionals $\left\{\varphi_{s}(\cdot)\right\}_{s=1}^{\infty}$, there exist an adjoint biorthogonal [9] system of functionals $\left\{\gamma_{j}(\cdot)\right\}_{j=1}^{\mu} \subset \mathbf{B}_{1}^{*}, \gamma_{j}(\cdot)=\operatorname{col}\left(\gamma_{j}^{(1)}(\cdot), \gamma_{j}^{(2)}(\cdot), \gamma_{j}^{(3)}(\cdot), \ldots\right)$, and an adjoint biorthogonal complete system of elements $\left\{\psi_{k}\right\}_{k=1}^{\infty} \subset \mathbf{B}_{2}, \psi_{k}=\operatorname{col}\left(\psi_{k}^{(1)}, \psi_{k}^{(2)}, \psi_{k}^{(3)}, \ldots\right)$.

The functionals $\left\{\gamma_{j}(\cdot)\right\}_{j=1}^{\mu}$ and $\left\{\varphi_{s}(\cdot)\right\}_{s=1}^{\infty}$ defined on the subspaces $N(L) \subset \mathbf{B}_{1}$ and $Y \subset \mathbf{B}_{2}$ (according to the Hahn-Banach theorem) can be extended to the spaces $\mathbf{B}_{1}$ and $\mathbf{B}_{2}$, respectively, with preservation of norm.

Let

$$
\begin{gathered}
X=\left(f_{1}, f_{2}, \ldots, f_{\mu}\right), \\
\Gamma(\cdot)=\left(\gamma_{1}(\cdot), \gamma_{2}(\cdot), \ldots, \gamma_{\mu}(\cdot)\right)^{T}, \\
\Phi(\cdot)=\left(\varphi_{1}(\cdot), \varphi_{2}(\cdot), \ldots, \varphi_{k}(\cdot), \ldots\right)^{T}, \\
\Psi=\left(\psi_{1}, \psi_{2}, \ldots, \psi_{k}, \ldots\right)
\end{gathered}
$$

denote, respectively, $\infty \times \mu, \mu \times \infty, \infty \times \infty$, and $\infty \times \infty$ matrices; furthermore, $\Gamma(X)=E_{\mu}$ and $\Phi(\Psi)=E_{\infty}$, where $E_{\mu}$ and $E_{\infty}$ are the identity matrices.

Taking notation (4) into account, we construct a projection operator $\mathcal{P}_{N(L)}: \mathbf{B}_{1} \rightarrow N(L)$ according to the formula

$$
\mathcal{P}_{N(L)}(\cdot)=X \Gamma(\cdot), \quad \mathcal{P}_{N(L)}: \mathbf{B}_{1} \rightarrow \mathbf{B}_{1} .
$$

For the construction of a projection operator $\mathcal{P}_{Y}: \mathbf{B}_{2} \rightarrow \mathbf{B}_{2}$, we consider the sequence of projectors

$$
\mathcal{P}_{Y^{(j)}}(\cdot)=\Psi_{j} \Phi_{j}(\cdot)
$$

of the space $\mathbf{B}_{2}$ to the subspaces $Y_{j} \subset Y$ spanned by the elements $\left\{\psi_{k}\right\}_{k=1}^{j}$.

Lemma 1. The sequence (6) of projectors $\mathcal{P}_{Y^{(j)}}$ converges strongly (pointwise) to the projector

$$
\mathcal{P}_{Y}(\cdot)=\Psi \Phi(\cdot)=\lim _{j \rightarrow \infty} \Psi_{j} \Phi_{j}(\cdot), \quad \mathcal{P}_{Y}: \mathbf{B}_{2} \rightarrow Y,
$$

where $Y \subset \mathbf{B}_{2}$ is the infinite-dimensional space spanned by the complete system of elements $\left\{\psi_{s}\right\}_{s=1}^{\infty}$. 
Proof. Using the definition of strong convergence in the norm of the space $\mathbf{B}_{2}$ and the definition of the matrices $\Phi$ and $\Psi$, for $y \in Y \subset \mathbf{B}_{2}$ we get

$$
\left\|\mathcal{P}_{Y} y-\mathcal{P}_{Y_{j}} y\right\|=\left\|\sum_{\xi=1}^{\infty} \varphi_{\xi}(y) \psi_{\xi}-\sum_{\xi=1}^{j} \varphi_{\xi}(y) \psi_{\xi}\right\|=\left\|\sum_{\xi=j+1}^{\infty} \varphi_{\xi}(y) \psi_{\xi}\right\| \leq \sum_{\xi=j+1}^{\infty}\left\|\varphi_{\xi}(y) \psi_{\xi}\right\|
$$

The quantity

$$
\sum_{\xi=j+1}^{\infty}\left\|\varphi_{\xi}(y) \psi_{\xi}\right\|
$$

tends to zero as $j \rightarrow \infty$ as a remainder of the expansion

$$
\sum_{\xi=1}^{\infty} \varphi_{\xi}(y) \psi_{\xi}
$$

of an element $y \in Y$ in the system of elements $\left\{\psi_{\xi}\right\}_{\xi=1}^{\infty}$, which is convergent in norm. Since the functionals $\left\{\varphi_{j}\right\}_{j=1}^{\infty}$ can be extended to the entire space $\mathbf{B}_{2}$, with preservation of norm, we can conclude that

$$
\sum_{\xi=j+1}^{\infty}\left\|\varphi_{\xi}(y) \psi_{\xi}\right\| \rightarrow 0 \quad \text { as } \quad j \rightarrow \infty
$$

for any $y \in \mathbf{B}_{2}$.

The operators $\mathcal{P}_{N(L)}$ and $\mathcal{P}_{Y}$ are projectors, i.e., they satisfy the conditions $\mathcal{P}_{N(L)}^{2}=\mathcal{P}_{N(L)}$ and $\mathcal{P}_{Y}^{2}=\mathcal{P}_{Y}$. These projectors are bounded. The boundedness of the projector $\mathcal{P}_{N(L)}$ follows from its finite dimensionality, and the boundedness of the projector $\mathcal{P}_{Y}$ follows from the complementability of the image $R(L)$ of the operator $L$ [10].

The projectors $\mathcal{P}_{N(L)}$ and $\mathcal{P}_{Y}$ divide the spaces $\mathbf{B}_{1}$ and $\mathbf{B}_{2}$ into direct topological sums of closed subspaces:

$$
\mathbf{B}_{1}=R\left(\mathcal{P}_{N(L)}\right) \oplus N\left(\mathcal{P}_{N(L)}\right), \quad \mathbf{B}_{2}=R\left(\mathcal{P}_{Y}\right) \oplus N\left(\mathcal{P}_{Y}\right)
$$

where $N\left(\mathcal{P}_{N(L)}\right)=X, R\left(\mathcal{P}_{N(L)}\right)=N(L), R\left(\mathcal{P}_{Y}\right)=Y$, and $N\left(\mathcal{P}_{Y}\right)=R(L)$.

Let us construct an isomorphism between the subspaces $N(L)$ and $Y_{1} \subset Y$. We compose the following matrices of $\mu$ rows and columns of the matrices $\Phi$ and $\Psi$ :

$$
\begin{gathered}
\bar{\Phi}(\cdot)=\left(\bar{\varphi}_{1}(\cdot), \bar{\varphi}_{2}(\cdot), \ldots, \bar{\varphi}_{\mu}(\cdot)\right)^{T}, \\
\bar{\Psi}=\left(\bar{\psi}_{1}, \bar{\psi}_{2}, \ldots, \bar{\psi}_{\mu}\right) .
\end{gathered}
$$

The matrix $\bar{\Psi}$ is composed of the system of elements $\left\{\bar{\psi}_{k}\right\}_{k=1}^{\mu} \subset\left\{\psi_{k}\right\}_{k=1}^{v}$ spanning the subspace $Y_{1}$, and the matrix $\bar{\Phi}$ is composed of functionals $\left\{\bar{\varphi}_{s}(\cdot)\right\}_{s=1}^{\mu} \subset\left\{\varphi_{s}(\cdot)\right\}_{s=1}^{\infty}$ that satisfy the relation $\bar{\varphi}_{s}\left(\bar{\psi}_{k}\right)=\delta_{s k}$, $s, k=1,2, \ldots, \mu$. 
We introduce the operators

$$
\begin{gathered}
\overline{\mathcal{P}}_{Y_{1}}(\cdot)=\bar{\Psi} \Gamma(\cdot), \quad(\cdot) \in \mathbf{B}_{1}, \\
\overline{\mathcal{P}}_{N(L)}(\cdot)=X \bar{\Phi}(\cdot), \quad(\cdot) \in \mathbf{B}_{2} .
\end{gathered}
$$

The operator $\overline{\mathcal{P}}_{Y_{1}}$ is an extension of an operator that performs an isomorphism of $N(L)$ onto $Y_{1}$ to the space $\mathbf{B}_{1}$, and $\overline{\mathcal{P}}_{N(L)}$ is an extension of the inverse of an isomorphic operator to the entire space $\mathbf{B}_{2}$.

Using (8), we define a projection operator $\mathcal{P}_{Y_{1}}: \mathbf{B}_{1} \rightarrow Y_{1} \subset Y$ by the relation

$$
\mathcal{P}_{Y_{1}}(\cdot)=\bar{\Psi} \bar{\Phi}(\cdot)
$$

This operator divides the subspace $Y$ into a direct topological sum of subspaces, namely,

$$
Y=Y_{1} \oplus Y_{2}
$$

where $Y_{2}=\mathcal{P}_{Y_{2}} \mathbf{B}_{2}=\left(\mathcal{P}_{Y}-\mathcal{P}_{Y_{1}}\right) \mathbf{B}_{2}$, and is bounded.

For the class of normally solvable $n$-normal operators, we prove a statement analogous to the Schmidt lemma.

Lemma 2. Let $L: \mathbf{B}_{1} \rightarrow \mathbf{B}_{2}$ be a linear, bounded, n-normal operator. Then the operator $\bar{L}=L+\overline{\mathcal{P}}_{Y_{1}}$ has a bounded left-inverse operator:

$$
\bar{L}_{l}^{-1}=\left(L+\overline{\mathcal{P}}_{Y_{1}}\right)_{l}^{-1}
$$

The general form of the left-inverse operators $\bar{L}_{l_{0}}^{-1}$ is given by the relation

$$
\bar{L}_{l}^{-1}=\bar{L}_{l_{0}}^{-1}\left(I_{\mathbf{B}_{2}}-\mathcal{P}_{Y_{2}}\right)
$$

Proof. Let $L$ be an $n$-normal operator. For the operator $\bar{L}$ to be left invertible, it is necessary and sufficient that the following conditions be satisfied [11]:

(i) $N(\bar{L})=\{0\}$;

(ii) the linear manifold $R(\bar{L})$ is a subspace that has a direct complement in $\mathbf{B}_{2}$.

Let us show that condition (i) is satisfied. Assume that there exists $x_{0} \neq 0, x_{0} \in \mathbf{B}_{1}$, such that

$$
\left(L+\overline{\mathcal{P}}_{Y_{1}}\right) x_{0}=L x_{0}+\bar{\Psi} \Gamma\left(x_{0}\right)=0 .
$$

It is obvious that $L x_{0} \in R(L)$. It follows from the definition of the operator $\overline{\mathcal{P}}_{Y_{1}}$ that $\overline{\mathcal{P}}_{Y_{1}} x_{0} \in Y_{1} \subset Y$. However, the subspaces $R(L)$ and $Y$ mutually complement one another to the entire space $\mathbf{B}_{2}$. Therefore, $R(L) \bigcap Y=\{0\}$, i.e., they have only one common (zero) element. Thus, $L x_{0}=0$ and $\overline{\mathcal{P}}_{Y_{1}} x_{0}=0$. This implies that $x_{0} \in N(L)$ and $x_{0} \in N\left(\overline{\mathcal{P}}_{Y_{1}}\right) \subset X$. However, the subspaces $N(L)$ and $X$ also mutually complement one another to the space $\mathbf{B}_{1}$. Therefore, $N(L) \bigcap X=\{0\}$. This implies that $x_{0}=0$. Thus, $N(L)=\{0\}$. 
Let us prove that condition (ii) is satisfied. The complementability of the image $R(\bar{L})$ in the space $\mathbf{B}_{2}$ follows from relation (11) and the complementability of the subspace $R(L)(2)$ :

$$
\mathbf{B}_{2}=R(L) \oplus Y_{1} \oplus Y_{2}=R(\bar{L}) \oplus Y_{2}
$$

Therefore, the operator $\bar{L}$ has a left inverse. The operator $\bar{L}$ maps the Banach space $\mathbf{B}_{1}$ bijectively to the subspace $\mathbf{B}_{2} \ominus Y_{2}$. Therefore, it follows from the Banach theorem [12] that the operator $\bar{L}_{l}^{-1}$ is bounded. It is known [11] that, in the general form, the left-inverse operators can be represented as follows: $\bar{L}_{l_{0}}^{-1}=\bar{L}_{l}^{-1} \mathcal{P}_{R(\bar{L})}$, where $\mathcal{P}_{R(\bar{L})}$ is a certain projector to the image $R(\bar{L})$ of the operator $\bar{L}$. It follows from (11) that the operator $I_{\mathbf{B}_{2}}-\mathcal{P}_{Y_{2}}$ possesses this property, i.e., $R\left(I_{\mathbf{B}_{2}}-\mathcal{P}_{Y_{2}}\right)=R(\bar{L})$. Thus, the general representation of left-inverse operators can be rewritten as follows:

$$
\bar{L}_{l_{0}}^{-1}=\bar{L}_{l}^{-1}\left(I_{\mathbf{B}_{2}}-\mathcal{P}_{Y_{2}}\right)
$$

Lemma 2 is proved.

Remark 1. If $\operatorname{dim} \operatorname{ker} L<\operatorname{dim} \operatorname{ker} L^{*}<\infty$, i.e., $L$ is a Noetherian operator of negative index, then Lemma 2 reduces to Lemma 2.4 in [4, p. 47].

Remark 2. If $\operatorname{dim} \operatorname{ker} L=\operatorname{dim} \operatorname{ker} L^{*}=n<\infty$, i.e, $L$ is a Fredholm operator of nonzero index, then Lemma 2 reduces to the Schmidt lemma [3, p. 340].

We now establish several properties of and relations between the left-inverse operator $\bar{L}_{l_{0}}^{-1}$, projectors (5) and (7), and operators (9).

Lemma 3. The operator $\bar{L}_{l_{0}}^{-1}$ satisfies the following relations:

$$
\begin{aligned}
& \text { (a) } \mathcal{P}_{N(L)} \bar{L}_{l_{0}}^{-1}=\overline{\mathcal{P}}_{N(L)}, \\
& \left(a_{2}\right) \quad L \bar{L}_{l_{0}}^{-1}=I_{\mathbf{B}_{2}}-\mathcal{P}_{Y}, \\
& \left(a_{3}\right) \quad \bar{L}_{l_{0}}^{-1} \mathcal{P}_{Y}=\overline{\mathcal{P}}_{N(L)}, \\
& \left(a_{4}\right) \bar{L}_{l_{0}}^{-1} L=I_{\mathbf{B}_{1}}-\mathcal{P}_{N(L)},
\end{aligned}
$$

where $I_{\mathbf{B}_{1}}$ and $I_{\mathbf{B}_{2}}$ are the identity operators in the spaces $\mathbf{B}_{1}$ and $\mathbf{B}_{2}$, respectively.

Proof. It follows from the definition of the left-inverse operator $\bar{L}_{l_{0}}^{-1}$ that if it exists, then [11]

$$
\begin{gathered}
\bar{L} \bar{L}_{l_{0}}^{-1}=I_{\mathbf{B}_{2}}-\mathcal{P}_{Y_{2}}, \\
\bar{L}_{l_{0}}^{-1} \bar{L}=I_{\mathbf{B}_{1}} .
\end{gathered}
$$

Since

$$
\overline{\mathcal{P}}_{N(L)} L=0, \quad \overline{\mathcal{P}}_{N(L)} \overline{\mathcal{P}}_{Y_{1}}=\mathcal{P}_{N(L)},
$$


acting by the operator $\bar{L}$ on both sides of equality (a 1$)$ in (12) from the right we obtain the identity

$$
\mathcal{P}_{N(L)}=\mathcal{P}_{N(L)} I_{\mathbf{B}_{1}}=\mathcal{P}_{N(L)} \bar{L}_{l_{0}}^{-1} \bar{L} \equiv \overline{\mathcal{P}}_{N(L)}\left(L+\overline{\mathcal{P}}_{Y_{1}}\right)=\overline{\mathcal{P}}_{N(L)} L+\overline{\mathcal{P}}_{N(L)} \overline{\mathcal{P}}_{Y_{1}}=\mathcal{P}_{N(L)},
$$

which proves this relation.

Since $\mathcal{P}_{Y} \overline{\mathcal{P}}_{Y_{1}}=\overline{\mathcal{P}}_{Y_{1}}$ and $\mathcal{P}_{Y}(L x)=0$ because $L x \in R(L)$, acting by the operator $L+\overline{\mathcal{P}}_{Y_{1}}$ on both sides of equality $\left(\mathrm{a}_{2}\right)$ in $(12)$ from the right we obtain the identity

$$
L=L I_{\mathbf{B}_{1}}=L \bar{L}_{l_{0}}^{-1} \bar{L} \equiv\left(I_{\mathbf{B}_{2}}-\mathcal{P}_{Y}\right)\left(L+\overline{\mathcal{P}}_{Y_{1}}\right)=L+\overline{\mathcal{P}}_{Y_{1}}-\mathcal{P}_{Y} L-\mathcal{P}_{Y} \overline{\mathcal{P}}_{Y_{1}}=L+\overline{\mathcal{P}}_{Y_{1}}-\overline{\mathcal{P}}_{Y_{1}}=L
$$

which proves this relation.

Further, since $\mathcal{P}_{Y}^{2}=\mathcal{P}_{Y}$ and $L \overline{\mathcal{P}}_{N(L)}=0$, acting by the operator $L$ on both sides of equality (a) in (12) from the left and using equality $\left(\mathrm{a}_{2}\right)$ we obtain the identity

$$
0=\left(I_{\mathbf{B}_{2}}-\mathcal{P}_{Y}\right) \mathcal{P}_{Y}=L \bar{L}_{l_{0}}^{-1} \mathcal{P}_{Y} \equiv L \overline{\mathcal{P}}_{N(L)}=0,
$$

which proves equality $\left(\mathrm{a}_{3}\right)$ in (12).

Acting by the operator $\bar{L}_{l_{0}}^{-1}$ on both sides of equality $\left(a_{4}\right)$ from the right and using equalities $\left(a_{1}\right)-\left(a_{3}\right)$ in (12), we obtain the identity

$$
\begin{aligned}
\bar{L}_{l_{0}}^{-1}-\overline{\mathcal{P}}_{N(L)} & =\bar{L}_{l_{0}}^{-1} I_{\mathbf{B}_{2}}-\bar{L}_{l_{0}}^{-1} \mathcal{P}_{Y}=\bar{L}_{l_{0}}^{-1} L \bar{L}_{l_{0}}^{-1} \\
& \equiv\left(I_{\mathbf{B}_{1}}-\mathcal{P}_{N(L)}\right) \bar{L}_{l_{0}}^{-1}=I_{\mathbf{B}_{1}} \bar{L}_{l_{0}}^{-1}-\mathcal{P}_{N(L)} \bar{L}_{l_{0}}^{-1}=\bar{L}_{l_{0}}^{-1}-\overline{\mathcal{P}}_{N(L)},
\end{aligned}
$$

which proves this relation.

Lemma 3 is proved.

Now let $L: \mathbf{B}_{1} \rightarrow \mathbf{B}_{2}$ be a linear, bounded, $d$-normal operator. In this case, the subspace $N(L)$ is infinitedimensional $(\mu=\infty)$, and the subspace $N\left(L^{*}\right)$ is finite-dimensional $(v<\infty)$. Assume that the space $\mathbf{B}_{1}$ has a basis. Then $N(L)$ also has a basis. Let $\left\{f_{i}\right\}_{i=1}^{\infty} \subset N(L)$ be a complete system of basis elements. The subspace $N\left(L^{*}\right)$ has a finite-dimensional basis $\left\{\varphi_{s}\right\}_{s=1}^{v} \subset N\left(L^{*}\right)$. For the elements $\left\{f_{i}\right\}_{i=1}^{\infty}$ and functionals $\left\{\varphi_{s}\right\}_{s=1}^{v}$, there exist an adjoint biorthogonal [9] system of functionals $\left\{\gamma_{j}\right\}_{j=1}^{\infty} \subset N^{*}(L) \subset \mathbf{B}_{1}^{*}$ and an adjoint biorthogonal complete system of elements $\left\{\psi_{k}\right\}_{k=1}^{v} \subset \mathbf{B}_{2}$. Each of the functionals $\left\{\gamma_{j}\right\}_{j=1}^{\infty}$ and $\left\{\varphi_{s}\right\}_{s=1}^{v}$ defined on the subspaces $N(L) \subset \mathbf{B}_{1}$ and $Y \subset \mathbf{B}_{2}$ (according to the Hahn-Banach theorem) can be extended to the spaces $\mathbf{B}_{1}$ and $\mathbf{B}_{2}$, respectively, with preservation of norm.

By analogy with (3), let

$$
\begin{gathered}
X=\left(f_{1}, f_{2}, \ldots, f_{s}, \ldots\right), \\
\Gamma(\cdot)=\left(\gamma_{1}(\cdot), \gamma_{2}(\cdot), \ldots, \gamma_{s}(\cdot), \ldots\right)^{T}, \\
\Phi(\cdot)=\left(\varphi_{1}(\cdot), \varphi_{2}(\cdot), \ldots, \varphi_{\nu}(\cdot)\right)^{T}, \\
\Psi=\left(\psi_{1}, \psi_{2}, \ldots, \psi_{\nu}\right)
\end{gathered}
$$


denote, respectively, $\infty \times \infty, \infty \times \infty, v \times \infty$, and $\infty \times v$ matrices; furthermore, $\Gamma(X)=E_{\infty}$ and $\Phi(\Psi)=E_{\nu}$, where $E_{\infty}$ and $E_{v}$ are the identity matrices.

To construct a projection operator $\mathcal{P}_{N(L)}: \mathbf{B}_{1} \rightarrow N(L)$, we define the sequence of projectors

$$
\mathcal{P}_{N^{(i)}(L)}(\cdot)=X_{i} \Gamma_{i}(\cdot), \quad i=1,2,3, \ldots,
$$

of the space $\mathbf{B}_{1}$ to the subspaces $N_{i}(L)$ of the null space $N(L)$.

Lemma 4. The sequence (13) of projectors $\mathcal{P}_{N^{(i)}(L)}$ converges strongly (pointwise) to the projector

$$
\mathcal{P}_{N(L)}(\cdot)=X \Gamma(\cdot)=\lim _{i \rightarrow \infty} X_{i} \Gamma_{i}(\cdot), \quad \mathcal{P}_{N(L)}: B_{1} \rightarrow N(L)
$$

The proof is analogous to the proof of Lemma 1.

We define a projection operator $\mathcal{P}_{Y}: \mathbf{B}_{2} \rightarrow Y$ of the space $\mathbf{B}_{2}$ to the subspace $Y$ as follows:

$$
\mathcal{P}_{Y}(\cdot)=\Psi \Phi(\cdot) .
$$

Consider the matrices

$$
\begin{gathered}
\bar{X}=\left(\bar{f}_{1}, \bar{f}_{2}, \ldots, \bar{f}_{v}\right), \\
\bar{\Gamma}(\cdot)=\left(\bar{\gamma}_{1}(\cdot), \bar{\gamma}_{2}(\cdot), \ldots, \bar{\gamma}_{v}(\cdot)\right)^{T} .
\end{gathered}
$$

The $\infty \times v$ matrix $\bar{X}$ is composed of arbitrary $v$ columns of the matrix $X$, and the $v \times \infty$ matrix $\bar{\Gamma}(\cdot)$ is composed of functionals of the matrix $\Gamma(\cdot)$ that satisfy the relation $\bar{\Gamma}(\bar{X})=E_{v}$, where $E_{v}$ is the identity matrix.

We introduce the operators

$$
\begin{gathered}
\overline{\mathcal{P}}_{Y}(\cdot)=\Psi \bar{\Gamma}(\cdot), \quad(\cdot) \in \mathbf{B}_{1}, \\
\overline{\mathcal{P}}_{N_{1}(L)}(\cdot)=\bar{X} \Phi(\cdot), \quad(\cdot) \in \mathbf{B}_{2} .
\end{gathered}
$$

The operator $\overline{\mathcal{P}}_{Y}: \mathbf{B}_{1} \rightarrow Y$ is an extension of an operator that realizes an isomorphism $N(L) \rightarrow Y$ to the entire space $\mathbf{B}_{1}$, and the operator $\overline{\mathcal{P}}_{N_{1}(L)}: \mathbf{B}_{2} \rightarrow N_{1}(L)$ is an extension of its inverse to the space $\mathbf{B}_{2}$.

Using (16), we define a projection operator $\mathcal{P}_{N_{1}(L)}: \mathbf{B}_{1} \rightarrow N_{1}(L) \subset N(L)$ as follows:

$$
\mathcal{P}_{N_{1}(L)}(\cdot)=\bar{X} \bar{\Gamma}(\cdot)
$$

This operator is bounded and divides the subspace $N(L)$ into a direct topological sum of subspaces, namely,

$$
N(L)=N_{1}(L) \oplus N_{2}(L), \quad N_{2}(L)=\mathcal{P}_{N_{2}(L)} \mathbf{B}_{1},
$$

where $\mathcal{P}_{N_{2}(L)}=\mathcal{P}_{N(L)}-\mathcal{P}_{N_{1}(L)}$ is a bounded projector.

For the class of normally solvable $d$-normal operators, we prove a statement analogous to the Schmidt lemma. 
Lemma 5. Let $L: \mathbf{B}_{1} \rightarrow \mathbf{B}_{2}$ be a linear, bounded, $d$-normal operator. Then the operator $\bar{L}=L+\overline{\mathcal{P}}_{Y}$ has a bounded right-inverse operator:

$$
\bar{L}_{r}^{-1}=\left(L+\overline{\mathcal{P}}_{Y}\right)_{r}^{-1}
$$

The general form of the right-inverse operators $\bar{L}_{r_{0}}^{-1}$ is given by the relation

$$
\bar{L}_{r_{0}}^{-1}=\left(I_{\mathbf{B}_{1}}-\mathcal{P}_{N_{2}(L)}\right) \bar{L}_{r}^{-1}
$$

The proof is analogous to the proof of Lemma 2 and is not presented here.

Remark 3. If $\operatorname{dim} \operatorname{ker} L^{*}<\operatorname{dim} \operatorname{ker} L<\infty$, i.e., $L$ is a Noetherian operator of positive index, then Lemma 5 reduces to Lemma 2.4 in [4, p. 47].

For the right-inverse operator $\bar{L}_{r_{0}}^{-1}$, the following lemma is true (this lemma is analogous to Lemma 3 and is presented here without proof):

Lemma 6. The operator $\bar{L}_{r_{0}}^{-1}$ satisfies the following relations:

$$
\begin{aligned}
& \text { (a) } \mathcal{P}_{N(L)} \bar{L}_{r_{0}}^{-1}=\overline{\mathcal{P}}_{N_{1}(L)}, \\
& \left(a_{2}\right) \quad L \bar{L}_{r_{0}}^{-1}=I_{\mathbf{B}_{2}}-\mathcal{P}_{Y}, \\
& \left(a_{3}\right) \bar{L}_{r_{0}}^{-1} \mathcal{P}_{Y}=\overline{\mathcal{P}}_{N_{1}(L)}, \\
& \left(a_{4}\right) \bar{L}_{r_{0}}^{-1} L=I_{\mathbf{B}_{1}}-\mathcal{P}_{N(L)},
\end{aligned}
$$

where $I_{\mathbf{B}_{1}}$ and $I_{\mathbf{B}_{2}}$ are the identity operators in the spaces $\mathbf{B}_{1}$ and $\mathbf{B}_{2}$, respectively.

Using the lemmas proved above, we can propose constructions of generalized inverse operators for $n$-normal and $d$ - normal operators.

We now formulate the main result of this paper.

Theorem 1. Let $L$ be a linear, bounded, n-normal operator whose image $R(L)$ is complementable in the Banach space $\mathbf{B}_{2}$. Then

$$
L^{-}=\bar{L}_{l_{0}}^{-1}-\overline{\mathcal{P}}_{N(L)}
$$

is a bounded generalized inverse operator for $L$.

Proof. It is necessary and sufficient to verify that $L^{-}$possesses the properties that define a generalized inverse operator [13], namely,

$$
L^{-}=L^{-} L L^{-}, \quad L=L L^{-} L
$$

To this end, we first show that

$$
L L^{-}=I_{\mathbf{B}_{2}}-\mathcal{P}_{Y}, \quad L^{-} L=I_{\mathbf{B}_{1}}-\mathcal{P}_{N(L)}
$$


Using equality ( $\left.\mathrm{a}_{2}\right)$ from (12) and representation (19), we get

$$
L L^{-}=L\left(\bar{L}_{l_{0}}^{-1}-\overline{\mathcal{P}}_{N(L)}\right)=L \bar{L}_{l_{0}}^{-1}-L \overline{\mathcal{P}}_{N(L)}=I_{\mathbf{B}_{2}}-\mathcal{P}_{Y}
$$

Using the relation

$$
\overline{\mathcal{P}}_{N(L)}(L z)=0
$$

and equality $\left(\mathrm{a}_{4}\right)$ from $(12)$, we obtain

$$
L^{-} L=\left(\bar{L}_{l_{0}}^{-1}-\overline{\mathcal{P}}_{N(L)}\right) L=\bar{L}_{l_{0}}^{-1} L-\overline{\mathcal{P}}_{N(L)} L=I_{\mathbf{B}_{1}}-\mathcal{P}_{N(L)}
$$

Taking (11) into account, we verify property (20). We have

$$
\begin{gathered}
L L^{-} L=L\left(I_{\mathbf{B}_{1}}-\mathcal{P}_{N(L)}\right)=L-L \mathcal{P}_{N(L)}=L, \\
L^{-} L L^{-}=\left(I_{\mathbf{B}_{1}}-\mathcal{P}_{N(L)}\right) L^{-}=L^{-}-\mathcal{P}_{N(L)} L^{-}=L^{-}
\end{gathered}
$$

because

$$
\mathcal{P}_{N(L)} L^{-}=\mathcal{P}_{N(L)} \bar{L}_{l_{0}}^{-1}-\mathcal{P}_{N(L)} \overline{\mathcal{P}}_{N(L)}=\overline{\mathcal{P}}_{N(L)}-\overline{\mathcal{P}}_{N(L)}=0
$$

The boundedness of the operator $L^{-}$follows from the boundedness of the operators $\bar{L}_{l_{0}}^{-1}$ and $\mathcal{P}_{N(L)}$.

The theorem is proved.

Remark 4. If a generalized inverse operator $L$ has a finite-dimensional kernel and a finite-dimensional cokernel, i.e., if it is a Noetherian operator, then construction (19) reduces to construction 2.14 in [4, p. 53].

Using Lemma 5, we formulate a theorem that gives a construction of a generalized inverse of a $d$-normal operator.

Theorem 2. Let $L$ be a linear, bounded, $d$-normal operator whose kernel $N(L)$ is complementable in the Banach space $\mathbf{B}_{1}$. Then

$$
L^{-}=\bar{L}_{r_{0}}^{-1}-\overline{\mathcal{P}}_{N_{1}(L)}
$$

is a bounded generalized inverse operator for $L$.

Proof. The theorem is proved by the verification of relations (20), which define a generalized inverse operator. The proof is analogous to that of Theorem 1 .

Using Theorems 1 and 2 and constructions (19) and (22), we can find a solution of the operator equation (1) with linear, bounded, $n$-normal (or $d$-normal) operator $L$ in explicit form. The general solution of Eq. (1) is the direct sum of the general solution $\tilde{x}$ of the homogeneous equation $L x=0$ corresponding to Eq. (1) and the particular solution $\bar{x}=L^{-} y$ of the inhomogeneous equation (1). It follows from the definition of a projector to the null space $N(L)$ of the operator $L$ that the general solution of the homogeneous equation can be represented in the form

$$
\tilde{x}=\mathcal{P}_{N(L)} \hat{x},
$$

where $\tilde{x} \in N(L) \subset \mathbf{B}_{1}$ and $\hat{x}$ is an arbitrary element of the space $\mathbf{B}_{1}$. 
Since the linear operator equation (1) is normally solvable, it is necessary and sufficient for its solvability that the following condition be satisfied [12]:

$$
\mathcal{P}_{N\left(L^{*}\right)} y=0,
$$

where $\mathcal{P}_{N\left(L^{*}\right)}$ is a projector to the null space of the operator $L^{*}$. Condition (23) guarantees that $y$ belongs to the image $R(L)$ of the operator $L$. Since $R(L)=N\left(\mathcal{P}_{Y}\right)$, relation (23) is equivalent to the condition

$$
\mathcal{P}_{Y} y=0 .
$$

This reasoning enables us to formulate the following theorem:

Theorem 3. Let $L$ be a linear, bounded, n-normal operator whose image $R(L)$ is complementable in the Banach space $\mathbf{B}_{2}$. The operator equation (1) with linear, bounded, n-normal operator is solvable for those and only those $y \in \mathbf{B}_{2}$ for which the following condition is satisfied:

$$
\mathcal{P}_{Y} y=0
$$

in this case, Eq. (1) has a $\mu$-parameter family of solutions representable in the form of a direct sum, namely,

$$
x=\tilde{x}+\bar{x}=\mathcal{P}_{N(L)} \hat{x}+L^{-} y,
$$

where the first term is the general solution of the corresponding homogeneous equation and the second term is a particular solution of the operator equation (1).

Proof. Substituting solution (25) into the original equation (1) and taking into account the first relation in (21) and condition (24), we obtain

$$
L x=L \mathcal{P}_{N(L)} \hat{x}+L L^{-} y=L L^{-} y=\left(I_{\mathbf{B}_{2}}-\mathcal{P}_{Y}\right) y=I_{\mathbf{B}_{2}} y-\mathcal{P}_{Y} y=I_{\mathbf{B}_{2}} y=y .
$$

With regard for the definition (5) of the operator $\mathcal{P}_{N(L)}$, we represent a solution of Eq. (1) in the form

$$
x=\mathcal{P}_{N(L)} \hat{x}+L^{-} y=X \Gamma(\hat{x})+L^{-} y=X c+L^{-} y,
$$

where $c=\Gamma(\hat{x})$ is an arbitrary constant vector from the space $R^{\mu}$.

Since the projector $\mathcal{P}_{Y}$ is infinite-dimensional, a linear equation with $n$-normal operator is solvable if and only if $y \in \mathbf{B}_{2}$ satisfies infinitely many linearly independent conditions.

The following theorem is true for linear equations with $d$-normal operator:

Theorem 4. Let $L$ be a linear, bounded, d-normal operator whose kernel $N(L)$ is complementable in the Banach space $\mathbf{B}_{1}$. The operator equation (1) with linear, bounded, $d$-normal operator is solvable for those and only those $y \in \mathbf{B}_{2}$ for which condition (24) is satisfied; in this case, Eq. (1) has an infinite-dimensional family of solutions representable in the form of a direct sum, namely,

$$
x=\tilde{x}+\bar{x}=\mathcal{P}_{N(L)} \hat{x}+L^{-} y,
$$

where the first term is the general solution of the corresponding homogeneous equation and the second term is a particular solution of the operator equation (1). 
With regard for the definition of the operator $\mathcal{P}_{N(L)}$, we represent a solution of Eq. (1) in the form

$$
x=\mathcal{P}_{N(L)} \hat{x}+L^{-} y=X \Gamma(\hat{x})+L^{-} y=X c+L^{-} y,
$$

where $c=\Gamma(\hat{x})$ is an arbitrary constant vector from a Banach space of number sequences $c=\left\{c_{1}, c_{2}, c_{3}, \ldots\right\}$ such that the series

$$
\sum_{i=1}^{\infty} f_{i} c_{i}
$$

is convergent.

Since the projector $\mathcal{P}_{Y}$ is finite-dimensional, a linear equation with $d$-normal operator is solvable if and only if $y \in \mathbf{B}_{2}$ satisfies finitely many linearly independent conditions.

Example 1. Let us find solvability conditions for the equation

$$
A x=y,
$$

where the $\infty \times n$ matrix $A$ has the form

$$
A=\left(\begin{array}{ccc}
\frac{1}{2} & 0 & \frac{1}{2} \\
0 & 0 & 0 \\
\frac{1}{4} & 0 & \frac{1}{4} \\
0 & 0 & 0 \\
\frac{1}{8} & 0 & \frac{1}{8} \\
0 & 0 & 0 \\
\frac{1}{16} & 0 & \frac{1}{16} \\
0 & 0 & 0 \\
\vdots & \vdots & \vdots
\end{array}\right),
$$

and determine the general form of a solution of this equation. It is obvious that the elements $a_{i j}$ of the matrix $A$ satisfy the relation

$$
\sum_{i=1}^{\infty} \sum_{j=1}^{3} a_{i j}<\infty
$$

Therefore, $A$ is a linear bounded operator that maps $R_{3}$ into $\boldsymbol{l}_{2}$ [14].

The operator $A$ is the simplest case of an $n$-normal operator that acts from the space $\mathbf{R}_{3}$ into the space $\boldsymbol{l}_{2}$ because $N(L)$ has two linearly independent basis vectors, namely 


$$
f_{1}=\left(\begin{array}{c}
1 \\
0 \\
-1
\end{array}\right) \quad \text { and } \quad f_{2}=\left(\begin{array}{l}
0 \\
1 \\
0
\end{array}\right)
$$

and $N\left(L^{*}\right)$ has infinitely many linearly independent basis vectors:

$$
\varphi_{1}=\left(\begin{array}{c}
-1 \\
0 \\
2 \\
0 \\
0 \\
0 \\
0 \\
0 \\
\vdots
\end{array}\right), \quad \varphi_{2}=\left(\begin{array}{c}
0 \\
1 \\
0 \\
0 \\
0 \\
0 \\
0 \\
0 \\
\vdots
\end{array}\right), \quad \varphi_{3}=\left(\begin{array}{c}
0 \\
0 \\
2 \\
0 \\
-4 \\
0 \\
0 \\
0 \\
\vdots
\end{array}\right),
$$

$$
\varphi_{4}=\left(\begin{array}{c}
0 \\
0 \\
0 \\
1 \\
0 \\
0 \\
0 \\
0 \\
\vdots
\end{array}\right), \quad \varphi_{5}=\left(\begin{array}{c}
0 \\
0 \\
0 \\
0 \\
-4 \\
0 \\
8 \\
0 \\
\vdots
\end{array}\right), \ldots
$$

Let us construct the generalized inverse of the operator $A$.

The matrices $X$ and $\Gamma$ composed of the basis vectors $f_{i}$ and $\gamma_{j}$ have the form

$$
X=\left(\begin{array}{cc}
1 & 0 \\
0 & 1 \\
-1 & 0
\end{array}\right), \quad \Gamma=\left(\begin{array}{ll}
1 & 0 \\
0 & 1 \\
0 & 0
\end{array}\right)^{T}, \quad \Gamma(X)=E_{3}
$$

Then we construct a projection operator $\mathcal{P}_{N(A)}: \mathbf{R}_{3} \rightarrow N(A)$ according to the formula

$$
\mathcal{P}_{N(A)}=X \Gamma=\left(\begin{array}{ccc}
1 & 0 & 0 \\
0 & 1 & 0 \\
-1 & 0 & 0
\end{array}\right), \quad \mathcal{P}_{N(A)}: \mathbf{R}_{3} \rightarrow \mathbf{R}_{3} .
$$

The matrices $\Phi$ and $\Psi$ composed of the basis vectors $\varphi_{s}$ and $\psi_{k}$ have the form 


$$
\Phi=\left(\begin{array}{cccccccccc}
-1 & 0 & 0 & 0 & 0 & 0 & 0 & 0 & \ldots \\
0 & 1 & 0 & 0 & 0 & 0 & 0 & 0 & \ldots \\
2 & 0 & 2 & 0 & 0 & 0 & 0 & 0 & \ldots \\
0 & 0 & 0 & 1 & 0 & 0 & 0 & 0 & \ldots \\
0 & 0 & -4 & 0 & -4 & 0 & 0 & 0 & \ldots \\
0 & 0 & 0 & 0 & 0 & 1 & 0 & 0 & \ldots \\
0 & 0 & 0 & 0 & 8 & 0 & 8 & 0 & \ldots \\
0 & 0 & 0 & 0 & 0 & 0 & 0 & 1 & \ldots \\
-1 & 0 & 0 & 0 & 0 & 0 & 0 & 0 & \ldots \\
0 & 1 & 0 & 0 & 0 & 0 & 0 & 0 & \ldots & \\
0 & 0 & 0 & 0 & 0 & 0 & 0 & 0 & \ldots & \\
0 & 0 & 0 & 1 & 0 & 0 & 0 & 0 & \ldots & \\
0 & 0 & -\frac{1}{4} & 0 & 0 & 0 & 0 & 0 & \ldots & \\
0 & \ldots & \ldots & \ldots & \ldots & \ldots & \ldots & \ldots & \ldots & \ldots
\end{array}\right), \quad \Phi(\Psi)=E_{\infty} .
$$

We construct a projection operator $\mathcal{P}_{Y}: \boldsymbol{l}_{2} \rightarrow Y$ according to formula (7)

$$
\mathcal{P}_{Y}=\Psi \Phi=\left(\begin{array}{ccccccccc}
1 & 0 & -2 & 0 & 0 & 0 & 0 & 0 & \ldots \\
0 & 1 & 0 & 0 & 0 & 0 & 0 & 0 & \ldots \\
0 & 0 & 0 & 0 & 0 & 0 & 0 & 0 & \ldots \\
0 & 0 & 0 & 1 & 0 & 0 & 0 & 0 & \ldots \\
0 & 0 & -\frac{1}{2} & 0 & 1 & 0 & 0 & 0 & \ldots \\
0 & 0 & 0 & 0 & 0 & 1 & 0 & 0 & \ldots \\
0 & 0 & -\frac{1}{4} & 0 & 0 & 0 & 1 & 0 & \ldots \\
0 & 0 & 0 & 0 & 0 & 0 & 0 & 1 & \ldots \\
\ldots & \ldots & \ldots & \ldots & \ldots & \ldots & \ldots & \ldots & \ldots
\end{array}\right), \quad \mathcal{P}_{Y}: l_{2} \rightarrow Y .
$$

Since the dimension of the null space $N(A)$ of the operator $A$ is equal to 2 , we define matrices $(8)$ as follows: 


$$
\begin{aligned}
& \bar{\Psi}=\left(\begin{array}{ccccccccc}
-1 & 0 & 0 & 0 & 0 & 0 & 0 & 0 & \ldots \\
0 & 1 & 0 & 0 & 0 & 0 & 0 & 0 & \ldots
\end{array}\right)^{T}, \\
& \bar{\Phi}=\left(\begin{array}{ccccccccc}
-1 & 0 & 2 & 0 & 0 & 0 & 0 & 0 & \ldots \\
0 & 1 & 0 & 0 & 0 & 0 & 0 & 0 & \ldots
\end{array}\right) .
\end{aligned}
$$

Then operators (9) take the form

$$
\begin{gathered}
\overline{\mathcal{P}}_{Y_{1}}=\bar{\Psi} \Gamma=\left(\begin{array}{ccc}
-1 & 0 & 0 \\
0 & 1 & 0 \\
0 & 0 & 0 \\
0 & 0 & 0 \\
\vdots & \vdots & \vdots
\end{array}\right), \\
\overline{\mathcal{P}}_{N(A)}=X \bar{\Phi}=\left(\begin{array}{cccccc}
-1 & 0 & 2 & 0 & 0 & \ldots \\
0 & 1 & 0 & 0 & 0 & \ldots \\
1 & 0 & -2 & 0 & 0 & \ldots
\end{array}\right) .
\end{gathered}
$$

We construct the operator $\bar{A}$ :

$$
\bar{A}=A+\overline{\mathcal{P}}_{Y_{1}}=\left(\begin{array}{ccc}
\frac{1}{2} & 0 & \frac{1}{2} \\
0 & 0 & 0 \\
\frac{1}{4} & 0 & \frac{1}{4} \\
0 & 0 & 0 \\
\frac{1}{8} & 0 & \frac{1}{8} \\
0 & 0 & 0 \\
\frac{1}{16} & 0 & \frac{1}{16} \\
0 & 0 & 0 \\
\vdots & \vdots & \vdots
\end{array}\right)+\left(\begin{array}{ccc}
-1 & 0 & 0 \\
0 & 1 & 0 \\
0 & 0 & 0 \\
0 & 0 & 0 \\
0 & 0 & 0 \\
0 & 0 & 0 \\
0 & 0 & 0 \\
0 & 0 & 0 \\
\vdots & \vdots & \vdots
\end{array}\right)=\left(\begin{array}{ccc}
-\frac{1}{2} & 0 & \frac{1}{2} \\
0 & 1 & 0 \\
\frac{1}{4} & 0 & \frac{1}{4} \\
0 & 0 & 0 \\
\frac{1}{8} & 0 & \frac{1}{8} \\
0 & 0 & 0 \\
\frac{1}{16} & 0 & \frac{1}{16} \\
0 & 0 & 0 \\
\vdots & \vdots & \vdots
\end{array}\right)
$$

The left inverse $\bar{A}_{l}^{-1}$ of the operator $\bar{A}$ has the form 


$$
\bar{A}_{l}^{-1}=\left(\begin{array}{cccccccccccc}
-1 & 0 & 2 & 0 & 1 & 0 & -2 & 0 & 1 & 0 & -2 & \ldots \\
0 & 1 & 0 & 1 & -1 & 0 & 2 & 0 & -1 & 0 & 2 & \ldots \\
1 & 0 & 2 & 0 & -8 & 0 & 16 & 0 & -32 & 0 & 64 & \ldots
\end{array}\right)
$$

To determine the general form of the left-inverse operators $\bar{A}_{l_{0}}^{-1}$, we construct the projector $I-\mathcal{P}_{Y_{2}}$. Using relation (10), we get

$$
\mathcal{P}_{Y_{1}}=\bar{\Psi} \bar{\Phi}=\left(\begin{array}{cc}
-1 & 0 \\
0 & 1 \\
0 & 0 \\
0 & 0 \\
0 & 0 \\
0 & 0 \\
\vdots & \vdots
\end{array}\right)\left(\begin{array}{cccccc}
-1 & 0 & 2 & 0 & 0 & \ldots \\
0 & 1 & 0 & 0 & 0 & \ldots
\end{array}\right)=\left(\begin{array}{cccccc}
-1 & 0 & 2 & 0 & 0 & \ldots \\
0 & 1 & 0 & 0 & 0 & \ldots \\
0 & 0 & 0 & 0 & 0 & \ldots \\
0 & 0 & 0 & 0 & 0 & \ldots \\
0 & 0 & 0 & 0 & 0 & \ldots \\
0 & 0 & 0 & 0 & 0 & \ldots \\
\ldots & \ldots & \ldots & \ldots & \ldots & \ldots
\end{array}\right) .
$$

Since $\mathcal{P}_{Y_{2}}=\mathcal{P}_{Y}-\mathcal{P}_{Y_{1}}$, we have

$$
I_{\mathbf{B}_{2}}-\mathcal{P}_{Y_{2}}=I_{\mathbf{B}_{2}}-\mathcal{P}_{Y}+\mathcal{P}_{Y_{1}}=\left(\begin{array}{ccccccc}
1 & 0 & 0 & 0 & 0 & 0 & \ldots \\
0 & 1 & 0 & 0 & 0 & 0 & \ldots \\
0 & 0 & 1 & 0 & 0 & 0 & \ldots \\
0 & 0 & 0 & 0 & 0 & 0 & \ldots \\
0 & 0 & \frac{1}{2} & 0 & 0 & 0 & \ldots \\
0 & 0 & 0 & 0 & 0 & 0 & \ldots \\
0 & 0 & \frac{1}{4} & 0 & 0 & 0 & \ldots \\
0 & 0 & 0 & 0 & 0 & 0 & \ldots \\
0 & 0 & \frac{1}{8} & 0 & 0 & 0 & \ldots \\
0 & 0 & 0 & 0 & 0 & 0 & \ldots \\
\ldots & \ldots & \ldots & \ldots & \ldots & \ldots & \ldots
\end{array}\right) .
$$

In the chosen basis, the general form of a left-inverse operator with respect to the projector $\mathcal{P}_{Y_{2}}$ is as follows:

$$
\bar{A}_{l_{0}}^{-1}=\bar{A}_{l}^{-1}\left(I_{\mathbf{B}_{2}}-\mathcal{P}_{Y_{2}}\right)=\left(\begin{array}{cccccccccc}
-1 & 0 & 2 & 0 & 0 & 0 & 0 & 0 & 0 & \ldots \\
0 & 1 & 0 & 0 & 0 & 0 & 0 & 0 & 0 & \ldots \\
1 & 0 & 2 & 0 & 0 & 0 & 0 & 0 & 0 & \ldots
\end{array}\right) .
$$

According to (20), the generalized inverse operator $A^{-}$has the form 


$$
A^{-}=\bar{A}_{l_{0}}^{-1}-\overline{\mathcal{P}}_{N(A)}=\left(\begin{array}{cccccccccc}
0 & 0 & 0 & 0 & 0 & 0 & 0 & 0 & 0 & \ldots \\
0 & 0 & 0 & 0 & 0 & 0 & 0 & 0 & 0 & \ldots \\
0 & 0 & 4 & 0 & 0 & 0 & 0 & 0 & 0 & \ldots
\end{array}\right)
$$

Then, by virtue of Theorem 4, Eq. (27) is solvable for those and only those $y \in \boldsymbol{l}_{2}$ that satisfy the condition

$$
\mathcal{P}_{Y} y=\left(\begin{array}{ccccccccc}
1 & 0 & -2 & 0 & 0 & 0 & 0 & 0 & \ldots \\
0 & 1 & 0 & 0 & 0 & 0 & 0 & 0 & \ldots \\
0 & 0 & 0 & 0 & 0 & 0 & 0 & 0 & \ldots \\
0 & 0 & 0 & 1 & 0 & 0 & 0 & 0 & \ldots \\
0 & 0 & -\frac{1}{2} & 0 & 1 & 0 & 0 & 0 & \ldots \\
0 & 0 & 0 & 0 & 0 & 1 & 0 & 0 & \ldots \\
0 & 0 & -\frac{1}{4} & 0 & 0 & 0 & 1 & 0 & \ldots \\
0 & 0 & 0 & 0 & 0 & 0 & 0 & 1 & \ldots \\
\ldots & \ldots & \ldots & \ldots & \ldots & \ldots & \ldots & \ldots & \ldots
\end{array}\right)\left(\begin{array}{c}
y_{1} \\
y_{2} \\
y_{3} \\
y_{4} \\
y_{5} \\
y_{6} \\
y_{7} \\
y_{8} \\
\ldots
\end{array}\right)=0 .
$$

For example, the vector

$$
y=\operatorname{col}\left(2 y_{3}, 0, y_{3}, 0, \frac{1}{2} y_{3}, 0, \frac{1}{4} y_{3}, 0, \ldots\right)
$$

satisfies this condition. Under this condition, the equation has a two-parameter family of solutions:

$$
\begin{aligned}
x & =\mathcal{P}_{N(L)} \hat{x}+L^{-} y=X \Gamma(\hat{x})+L^{-} y \\
& =\left(\begin{array}{ccc}
1 & 0 & 0 \\
0 & 1 & 0 \\
-1 & 0 & 0
\end{array}\right)\left(\begin{array}{c}
c_{1} \\
c_{2} \\
c_{3}
\end{array}\right)+\left(\begin{array}{llllllllll}
0 & 0 & 0 & 0 & 0 & 0 & 0 & 0 & 0 & \ldots \\
0 & 0 & 0 & 0 & 0 & 0 & 0 & 0 & 0 & \ldots \\
0 & 0 & 4 & 0 & 0 & 0 & 0 & 0 & 0 & \ldots
\end{array}\right)\left(\begin{array}{c}
2 y_{3} \\
0 \\
y_{3} \\
0 \\
\frac{1}{2} y_{3} \\
0 \\
1 \\
\frac{1}{4} \\
0 \\
0 \\
\vdots
\end{array}\right)=\left(\begin{array}{c}
c_{1} \\
c_{2} \\
-c_{1}
\end{array}\right)+\left(\begin{array}{c}
0 \\
0 \\
4 y_{3}
\end{array}\right) .
\end{aligned}
$$




\section{REFERENCES}

1. A. M. Samoilenko and Yu. V. Teplins'kyi, Elements of the Mathematical Theory of Evolution Equations in Banach Spaces [in Ukrainian], Institute of Mathematics, Ukrainian National Academy of Sciences, Kyiv (2008).

2. A. A. Boichuk and E. V. Panasenko, "Boundary-value problems for differential equations in a Banach space," Nelin. Kolyvannya, 12, No. 1, 16-19 (2009).

3. M. M. Vainberg and V. A. Trenogin, Theory of Branching of Solutions of Nonlinear Equations [in Russian], Nauka, Moscow (1969).

4. A. A. Boichuk, V. F. Zhuravlev, and A. M. Samoilenko, Generalized Inverse Operators and Noetherian Boundary-Value Problems [in Russian], Institute of Mathematics, Ukrainian National Academy of Sciences, Kiev (1995).

5. E. Schmidt, "Zur Theorie linearen und nichtlinearen Integralgleichungen. Teil 3. Über die Auflösungen der nichtlinearen Integralgleichungen und die Verzweigung ihrer Losungen," Math. Ann., No. 65, (1908).

6. Yu. M. Daletskii and M. G. Krein, Stability of Solutions of Differential Equations in a Banach Space [in Russian], Nauka, Moscow (1970).

7. S. G. Krein, Linear Differential Equations in a Banach Space [in Russian], Nauka, Moscow (1970).

8. L. A. Lyusternik and V. I. Sobolev, A Brief Course in Functional Analysis [in Russian], Vysshaya Shkola, Moscow (1982).

9. M. M. Grinblyum, "Biorthogonal systems in a Banach space," Dokl. Akad. Nauk SSSR, 47, No. 2, 79-82 (1945).

10. M. I. Kadets and B. S. Mityagin, “Complementable subspaces in Banach spaces,” Usp. Mat. Nauk, 28, Issue 6, 77-94 (1973).

11. I. Ts. Gokhberg and N. Ya. Krupnik, Introduction to the Theory of One-Dimensional Singular Integral Operators [in Russian], Shtiintsa, Kishinev (1973).

12. V. A. Trenogin, Functional Analysis [in Russian], Nauka, Moscow (1989).

13. A. A. Boichuk and A. M. Samoilenko, Generalized Inverse Operators and Fredholm Boundary-Value Problems, VSP, Utrecht (2004).

14. B. Z. Vulikh, Introduction to Functional Analysis [in Russian], Nauka, Moscow (1967). 